\title{
Upregulation of CCT3 promotes cervical cancer progression through FN1
}

\author{
LEI DOU $^{1}$ and XINXIN ZHANG ${ }^{2}$ \\ ${ }^{1}$ Department of Gynecology, The First Hospital of China Medical University; ${ }^{2}$ Department of \\ Discipline Inspection Commission, China Medical University, Shenyang, Liaoning 110001, P.R. China
}

Received January 20, 2021; Accepted September 3, 2021

DOI: $10.3892 / \mathrm{mmr} .2021 .12496$

\begin{abstract}
The mechanisms underlying cervical cancer progression have not yet been fully elucidated; thus, further investigations are required. Chaperonin containing TCP1 subunit 3 (CCT3) expression was found to be upregulated in several types of human cancer. However, the roles of CCT3 in cervical cancer remain poorly understood. Thus, the present study aimed to determine the roles of CCT3 in the progression of cervical squamous cell carcinoma and endocervical adenocarcinoma (CESC). For this purpose, the Tumor Immune Estimation Resource and Gene Expression Profiling Interactive Analysis databases were used to analyze the mRNA and protein expression levels of CCT3 in CESC samples. The effects of CCT3 on the proliferation and migration of CESC in vitro were determined using various experiments, including proliferation, Transwell and flow cytometric assays. The results revealed that CCT3 expression was significantly upregulated in CESC, which was associated with a poor prognosis. The silencing of CCT3 suppressed CESC cell proliferation, migration and invasiveness in vitro. Additionally, CCT3-knockdown promoted CESC cell apoptosis and cell cycle arrest, and suppressed fibronectin 1 (FN1) protein expression. Furthermore, rescue assays demonstrated that CCT3 promoted CESC proliferation and migration via FN1. In conclusion, the findings of the present study demonstrated that CCT3 is closely associated with the progression of CESC. Thus, CCT3 may be considered a novel, promising biomarker, and a possible therapeutic target for CESC.
\end{abstract}

Correspondence to: Dr Lei Dou, Department of Gynecology, The First Hospital of China Medical University, 155 Nanjing Street, Heping, Shenyang, Liaoning 110001, P.R. China

E-mail: doulei840416@163.com

Abbreviations: CESC, cervical squamous cell carcinoma and endocervical adenocarcinoma; CCT3, chaperone containing TCP1 subunit 3; FN1, fibronectin 1; GEO, Gene Expression Omnibus; TCGA, The Cancer Genome Atlas

Key words: CESC, CCT3, prognosis, proliferation, metastasis

\section{Introduction}

As indicated by global cancer statistics, cervical squamous cell carcinoma and endocervical adenocarcinoma (CESC) ranks as the fourth most common cause of cancer-related mortality in women $(1,2)$. On average, the 5-year survival rate of patients with CESC is $17 \%$ (3). Human papillomavirus (HPV) infection is considered the leading cause of CESC (4). However, it is suggested that other unknown mechanisms may be involved in CESC occurrence (4). It has been determined that inflammation plays an important role in tumor development (5). In addition to HPV infection, several other factors have been identified as crucial regulators of CESC progression, such as G-rich RNA sequence binding factor 1 and transmembrane emp24 domain-containing protein 5 (6). Thus far, the exact mechanisms underpinning CESC occurrence and progression remain elusive. Therefore, exploration of the mechanisms underlying the pathogenesis of CESC, and the development of novel treatment strategies, are essential.

Chaperone containing TCP1 (CCT) is a bicyclic chaperone complex comprising 8 unequal components (CCT1-CCT8) (7). CCT family members are of importance for actin and tubulin folding, which is necessary for cellular migration and cell cycle progression (8). Although CCT1 (9), CCT2 (10), CCT4 (11) and CCT8 (12) have been implicated in cellular proliferation, little is known of the function of CCT3 in tumorigenesis. Recent research in kidney cancer has identified CCT3 as a novel target and indicator of carcinoma, upstream of yes-associated protein and transcription factor CP2 (13). The expression of CCT3 was also found to be increased in hepatocellular carcinoma (HCC), which was associated with poor patient prognosis (14). In addition, CCT3 upregulation in thyroid papillary carcinoma (PTC) has been demonstrated to induce PTC cell proliferation, cell cycle arrest and apoptosis (15). In gastric cancer (GC), experimental CCT3-knockdown suppressed GC cellular proliferation and induced apoptosis. However, the exact role of CCT3 in the occurrence of CESC remains unclear.

The present study aimed to determine the correlation between CCT3 expression and CESC prognosis and the role of CCT3 in the progression of CESC. For this purpose, the Tumor Immune Estimation Resource and Gene Expression Profiling Interactive Analysis databases were used to analyze the mRNA and protein expression levels of CCT3 in CESC samples. The effects of CCT3 on the proliferation and migration of CESC 
in vitro were determined using various methods, including proliferation, Transwell and flow cytometric assays.

\section{Materials and methods}

Cell lines and culture. HeLa and SiHa cells were obtained from The Cell Bank of Type Culture Collection of the Chinese Academy of Sciences. All cells were cultured in RPMI-1640 medium (HyClone; Cytiva) supplemented with 10\% FBS (Gibco; Thermo Fisher Scientific, Inc.) at $37^{\circ} \mathrm{C}\left(5 \% \mathrm{CO}_{2}\right)$.

Construction of lentivirus expression cassette and transfection. To generate the cellular knockdown model, CCT3 short hairpin RNA (shRNA; 5'-GCTGTGAAGCTGCAGACTT-3') and shRNA negative control (shCtrl; 5'-TTCTCCGAACGTGTCACGT-3') were designed by Shanghai GeneChem Co., Ltd. A lentivirus expression cassette expressing CCT3 shRNA was cloned as described in a previous study (16). Briefly, after annealing, shRNA fragments were integrated into a lentiviral GV493 vector (hU6-MCS-CBh-GFP-IRES-puromycin; Shanghai GeneChem Co., Ltd.). To generate the overexpression model, the CCT3 and FN1 coding sequences were synthesized by Shanghai GeneChem Co., Ltd. and integrated into lentiviral GV492 (Ubi-MCS-3FLAG-CBh-gcGFP-IRES-puromycin). The empty GV492 vector was used as a negative control (OECtrl). A total of $4 \times 10^{5} 293 \mathrm{FT}$ cells per well were seeded into a 6 well plate, and transduced with the target gene construct and the envelope, packaging and recombinant lentiviral plasmids (3rd generation system; Cyagen Biosciences, Inc.) at $37^{\circ} \mathrm{C}$. After $48 \mathrm{~h}$, the cells were centrifuged at $10,000 \times \mathrm{g}$ for $4 \mathrm{~h}\left(4^{\circ} \mathrm{C}\right)$ and the virus-containing supernatant was harvested. HeLa and SiHa cells at the 3 rd passage were then infected (multiplicity of infection, 50) using $5 \mu \mathrm{g} / \mathrm{ml}$ Polybrene. The cells were cultured at $37^{\circ} \mathrm{C}$ for 3 days in RPMI-1640 medium containing $10 \% \mathrm{FBS}$ and $1 \mu \mathrm{g} / \mathrm{ml}$ puromycin (in order to select puromycin-resistant cells) and $0.25 \mu \mathrm{g} / \mathrm{ml}$ puromycin was used for maintenance. Similarly, HeLa and SiHa cells transfected with blank lentivirus were used to generate the controls (shCtrl and OECtrl). After 2 days, reverse transcription-quantitative PCR (RT-qPCR) was performed to confirm target gene expression. All transfections were conducted using Lipofectamine ${ }^{\circledR} 2000$ (Invitrogen; Thermo Fisher Scientific, Inc.).

Reverse transcription-quantitative PCR (RT-qPCR). Total RNA was extracted from cells using TRIzol ${ }^{\circledR}$ reagent (Invitrogen; Thermo Fisher Scientific, Inc.) and reverse transcribed into cDNA using the RevertAid First Strand cDNA Synthesis kit (Promega Corporation) following the manufacturer's instructions. qPCR was subsequently performed using $\mathrm{iQ}^{\mathrm{TM}}$ SYBR-Green Supermix (Bio-Rad Laboratories, Inc.) as previously described $(16,17)$. The qPCR reaction conditions were as follows: Initial denaturation at $95^{\circ} \mathrm{C}$ for $30 \mathrm{sec}$, followed by 40 cycles at $95^{\circ} \mathrm{C}$ for $5 \mathrm{sec}, 60^{\circ} \mathrm{C}$ for $10 \mathrm{sec}$ and $72^{\circ} \mathrm{C}$ for $30 \mathrm{sec}$. The specific primers were as follows: CCT3 forward, 5'-TCAGTCGGTGGTCATCTTTGG-3' and reverse, 5'-CCTCCAGGTATCTTTTCCACTCT-3'; and GAPDH forward, 5'-TGACTTCAACAGCGACACCCA-3' and reverse, 5'-CACCCTGTTGCTGTAGCCAAA-3'. Relative expression levels were calculated using the $2^{-\Delta \Delta C q}$ method (18).
Cell viability assay. Cell viability was determined using an MTT assay. HeLa and SiHa cells infected with CCT3 shRNA lentivirus and/or FN1 overexpression lentivirus were seeded into 96 -well plates $\left(2 \times 10^{3}\right.$ cells/well) and incubated at $37^{\circ} \mathrm{C}$ for 5 days. Following incubation, MTT (Sigma-Aldrich; Merck $\mathrm{KGaA}$ ) solution was added to each well, followed by incubation for a further $4 \mathrm{~h}$. The MTT solution was then aspirated and $100 \mu \mathrm{l}$ DMSO (Sigma-Aldrich; Merck KGaA) was added to dissolve the formazan crystals. The number of viable cells was counted using an automated microplate reader (Molecular Devices, LLC) at a wavelength of $570 \mathrm{~nm}$.

Celigo ${ }^{\circledR}$ adherent cell cytometry system. HeLa and $\mathrm{SiHa}$ cells infected with CCT3 shRNA lentivirus and/or the FN1 overexpression lentivirus were harvested in the logarithmic growth phase (Gibco; Thermo Fisher Scientific, Inc.), resuspended in complete medium and seeded into 96-well plates $\left(2 \times 10^{3}\right.$ cells/well). After plating, a Celigo Image Cytometer (Nexcelom) was used to evaluate the number of cells by scanning for green fluorescence daily, for 5 days at room temperature.

Flow cytometric analysis. HeLa and $\mathrm{SiHa}$ cells were transfected with CCT3 shRNA or the associated negative control. An apoptosis assay was performed using the Annexin V-FITC/ propidium iodide(PI) Apoptosis Detection kit (Nanjing KeyGen Biotech Co., Ltd.) following the manufacturer's protocol. The cells were analyzed using a flow cytometer (FACSCalibur; BD Biosciences) and FlowJo V10.0 (BD Biosciences). Cells were also stained using a PI staining kit (Nanjing KeyGen Biotech Co., Ltd.) and the cell cycle was evaluated by flow cytometry as previously described (16).

Cellular migration and invasion assays. Cellular invasion and migration assays were conducted using 24-well Transwell plates with or without Matrigel, respectively (BD Biosciences). For the migration assay, transfected cells $\left(1 \times 10^{5}\right)$ were seeded into the upper chamber without Matrigel. For the invasion assay, transfected cells $\left(1 \times 10^{5}\right)$ were seeded in the upper Matrigel-coated chamber without serum; for both assay types, the lower chamber was filled with culture medium supplemented with $15 \%$ FBS as a chemoattractant and cultured for $48 \mathrm{~h}$ in $37^{\circ} \mathrm{C}$. The migratory and invasive cells in the lower chamber were room temperature formalin-fixed for $30 \mathrm{~min}$, stained with crystal violet at room temperature for $20 \mathrm{~min}$ and washed with PBS. Cells were counted by Olympus CX35 light microscope.

Western blot analysis. Cells were harvested using an enzymatic digestion method. Subsequently, ice-cold lysis buffer $(50 \mathrm{mM}$ Tris, $150 \mathrm{mM} \mathrm{NaCl}, 0.5 \%$ EDTA and $0.5 \%$ NP-40) was added and cells were incubated for $20 \mathrm{~min}$ at $4^{\circ} \mathrm{C}$. Centrifugation was performed at $13,000 \mathrm{x} \mathrm{g}$ at $4^{\circ} \mathrm{C}$ for $15 \mathrm{~min}$. Total protein concentration was determined using a BCA Protein Quantification kit. Total protein (30 $\mu \mathrm{g} /$ lane) was separated via SDS-PAGE on a $10 \%$ gel and subsequently transferred to a PVDF membrane using the Bio-Rad Transfer System (Bio-Rad Laboratories, Inc.). Membranes were blocked with $5 \%$ non-fat dry milk for $1 \mathrm{~h}$ at room temperature and incubated with primary antibodies overnight at $4^{\circ} \mathrm{C}$ (Table SI). Following 

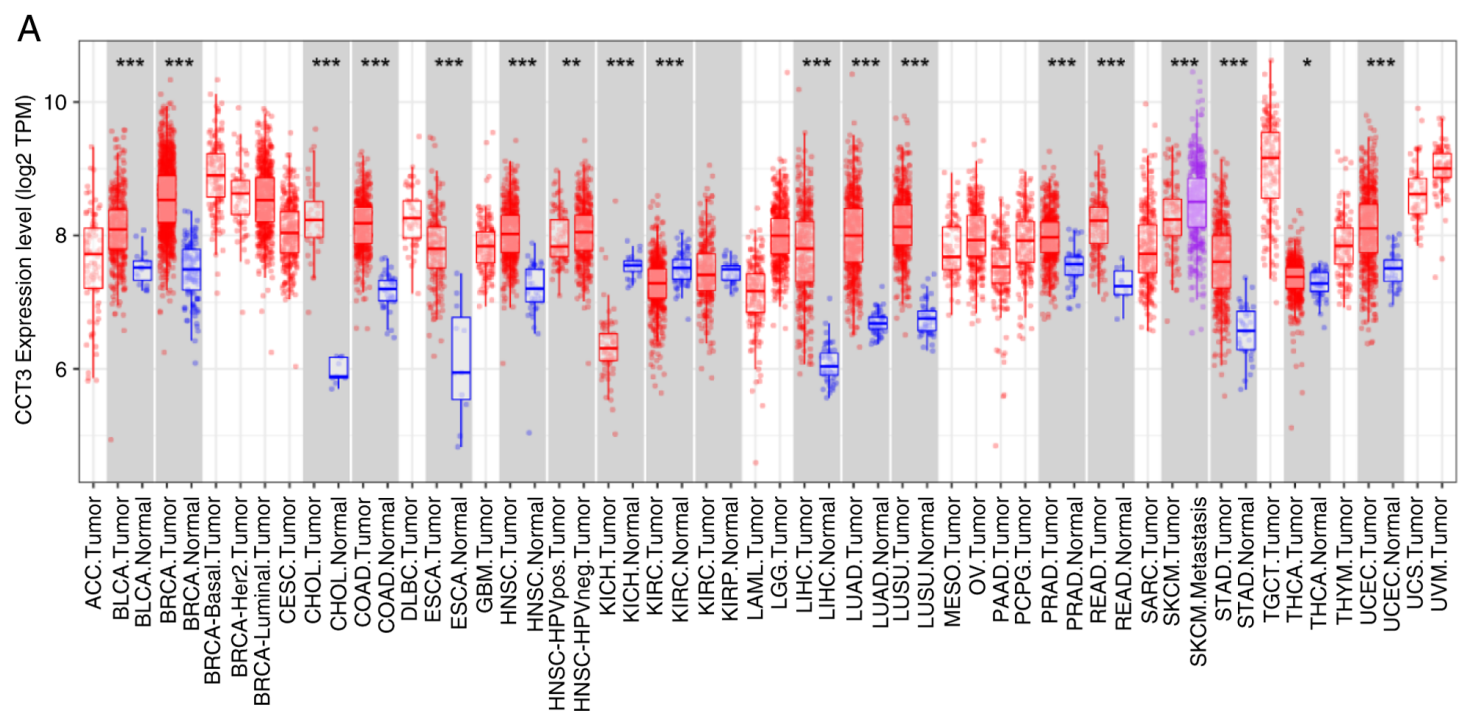

B

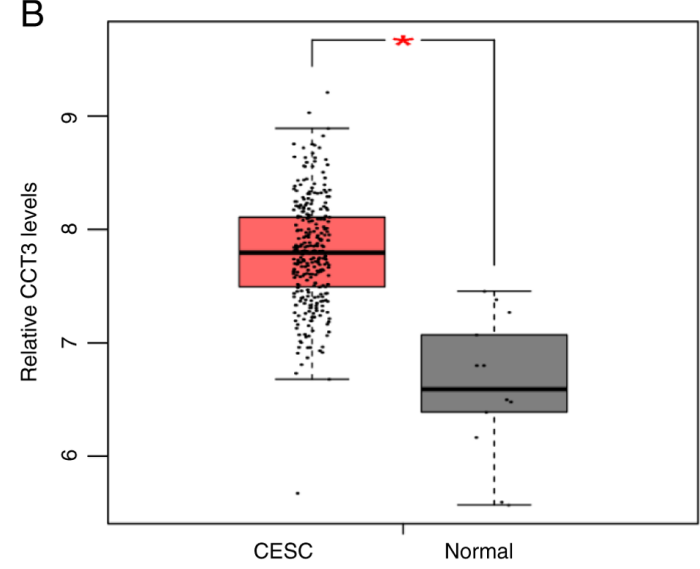

D

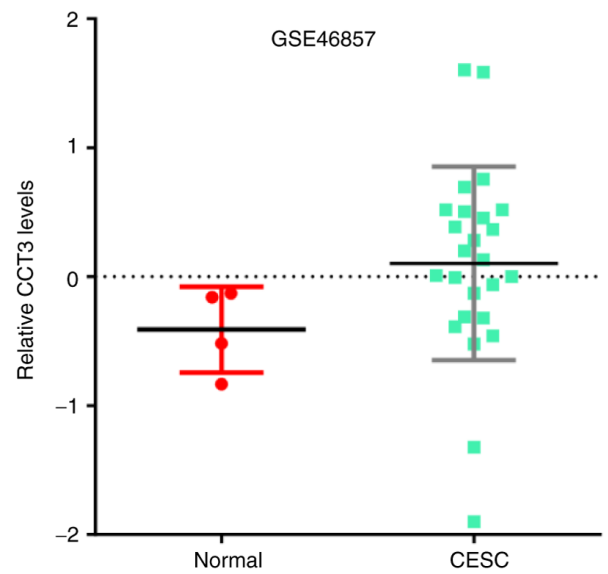

C

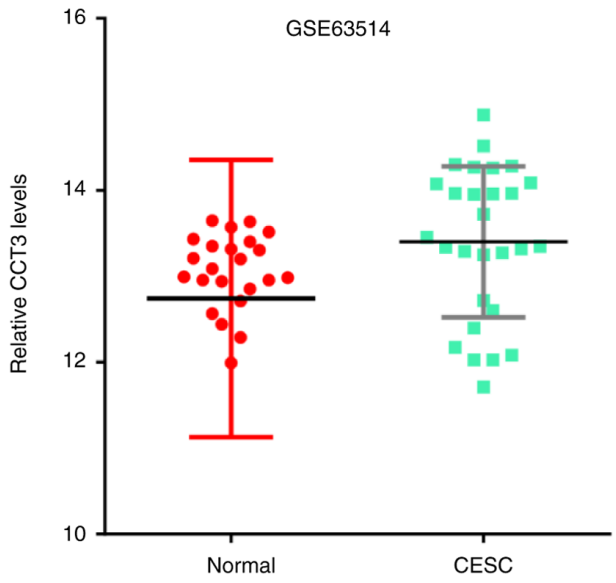

$\mathrm{E}$

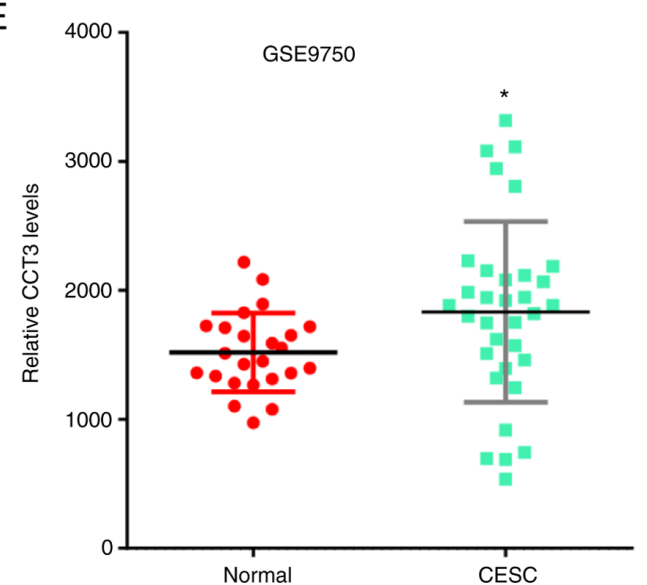

Figure 1. CCT3 expression profiles in GEO and The Cancer Genome Atlas datasets. (A) mRNA expression of CCT3 in 24 tumor types from the TIMER database. (B) CCT3 expression profiles in CESC tissues and normal tissues downloaded from the Gene Expression Profiling Interactive Analysis database. CCT3 expression was upregulated in CESC compared with the normal groups in the (C) GSE63514, (D) GSE46857 and (E) GSE9750 datasets from the GEO database. "P $<0.05$. CCT3, chaperonin containing TCP1 subunit 3; CESC, cervical squamous cell carcinoma and endocervical adenocarcinoma; GEO, Gene Expression Omnibus.

secondary antibody incubation for $1 \mathrm{~h}$ at room temperature (HRP-conjugated anti-rabbit and anti-mouse; both 1:3,000; cat. nos. 7074 and 7076, respectively; Cell Signaling Technology, Inc.), protein expression was determined using the Pierce ECL System (Thermo Fisher Scientific, Inc.). The protein band density was determined using ImageJ (version 1.53; National Institutes of Health).
Bioinformaticsanalysis.CCT3 mRNA expression in cancer and normal tissues was analyzed using Tumor Immune Estimation Resource (TIMER) 2.0 (http://timer.comp-genomics.org/) and the Gene Expression Profiling Interactive Analysis (GEPIA; http://gepia.cancer-pku.cn/) database and Gene Expression Omnibus (GEO) datasets [GSE63514, https:// www.ncbi.nlm.nih.gov/geo/query/acc.cgi?acc=GSE63514 (19), 
A

CESC

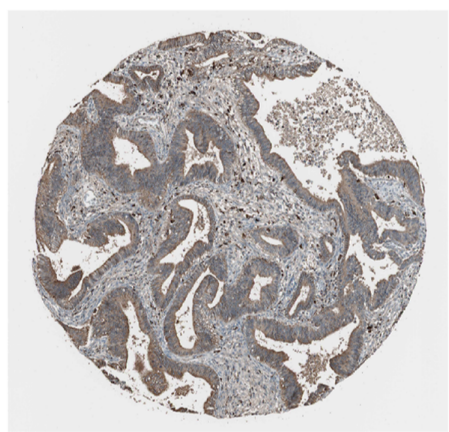

Normal

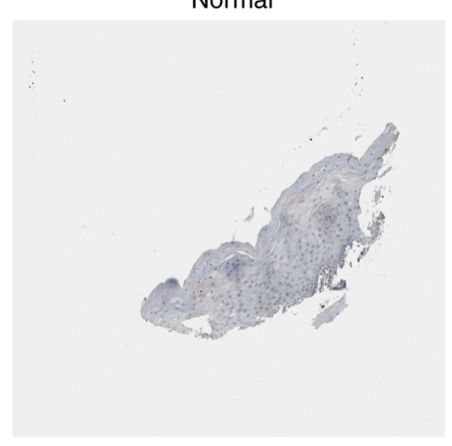

B

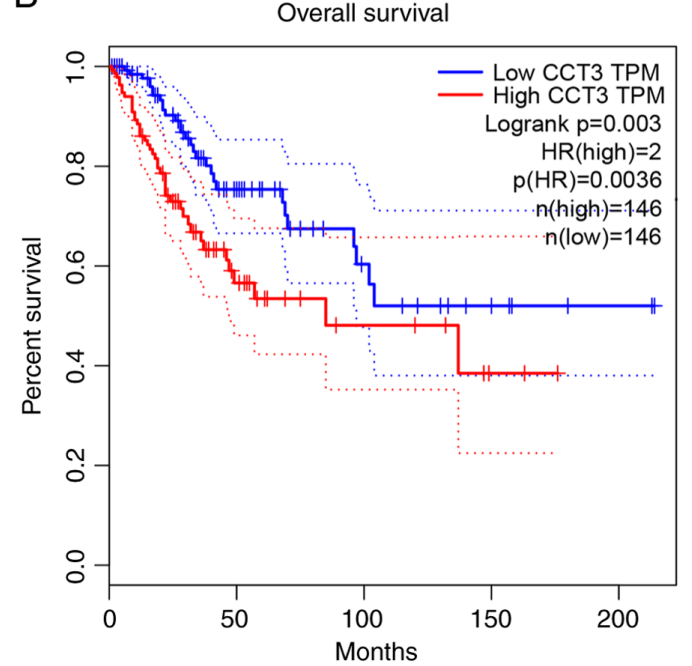

CESC

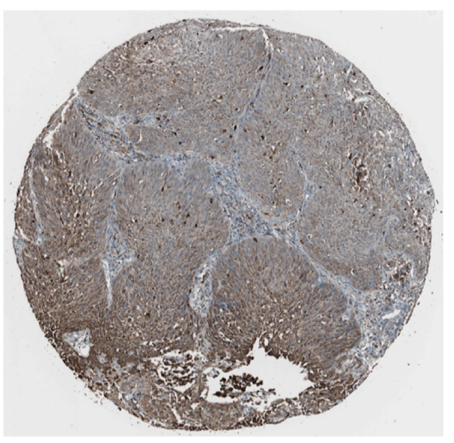

Normal

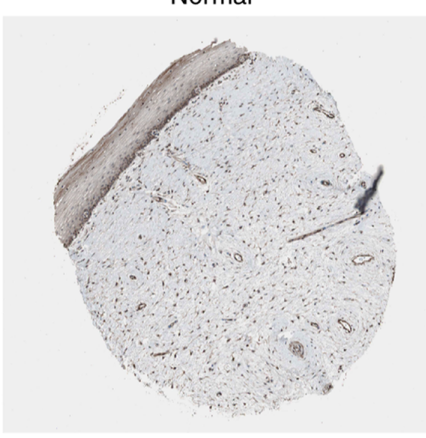

CESC

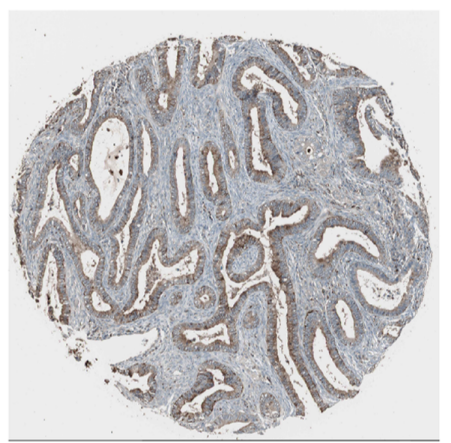

Normal

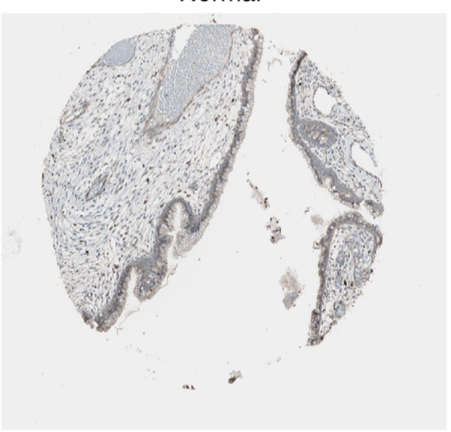

C Disease free survival

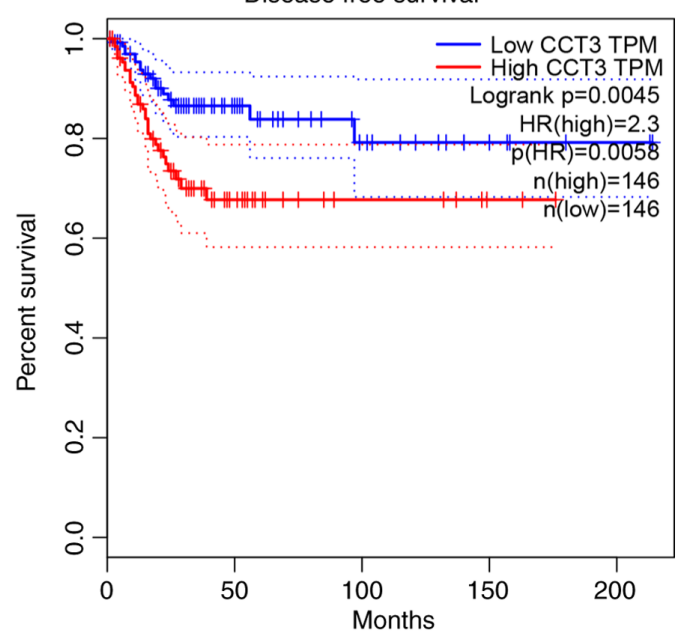

Figure 2. CCT3 is upregulated in CESC, which is associated with poor patient prognosis. (A) Data from the Protein Atlas database revealed that CCT3 protein expression was increased in CESC tumors compared with non-tumor tissues. Higher expression levels of CCT3 were associated with shorter (B) overall and (C) disease-free survival times in CESC. CCT3, chaperonin containing TCP1 subunit 3; CESC, cervical squamous cell carcinoma and endocervical adenocarcinoma.

GSE46857, https://www.ncbi.nlm.nih.gov/geo/query/acc. cgi?acc=GSE46857 (20) and GSE9750, https://www.ncbi.nlm. nih.gov/geo/query/acc.cgi?acc=GSE9750 (21) datasets]. The associations between patient overall survival and disease-free survival, and CCT3 mRNA expression levels were analyzed using TIMER 2.0 and the GEPIA database. The protein expression level of CCT3 in cancer and normal tissues was analyzed using the Protein Atlas database (https://www. proteinatlas.org/).

Statistical analysis. All data are presented as the mean \pm SD. Statistical differences between two groups were determined using the unpaired Student's t-test, and one-way ANOVA with Tukey's post hoc test was used to analyze the differences between multiple groups. $\mathrm{P}<0.05$ was considered to indicate a statistically significant difference. Overall survival was assessed using the Kaplan-Meier method. The log-rank test was applied to determine significant differences. Correlation between CCT3 and FN1 was calculated using Spearman's method. All statistical analyses were performed using GraphPad Prism 6 software (GraphPad Software, Inc.).

\section{Results}

Evaluation of CCT3 expression level in GEO and The Cancer Genome Atlas (TCGA) datasets. To determine the role of 

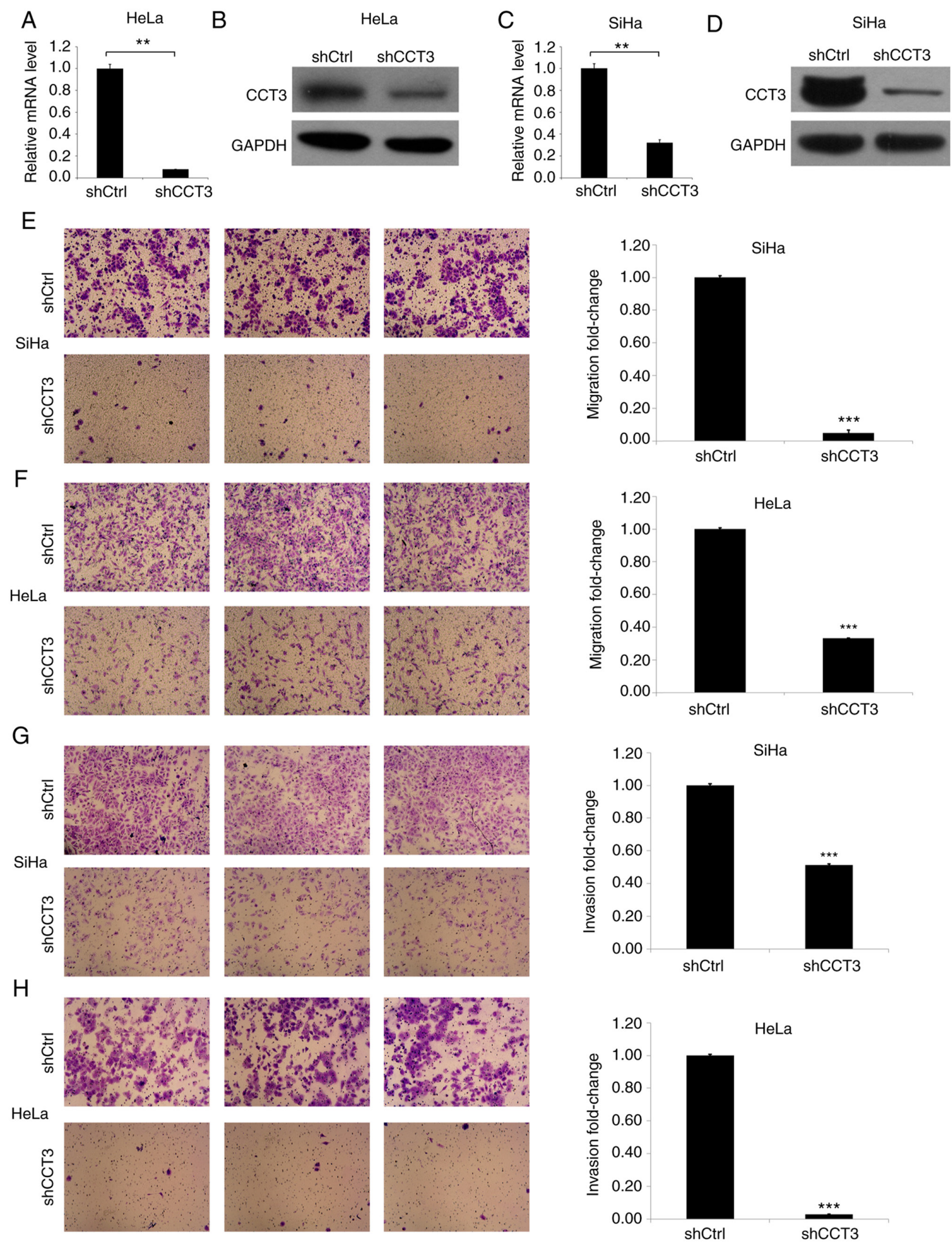

Figure 3. CCT3-knockdown inhibits CESC cell proliferation, invasiveness and migration in vitro. CCT3-knockdown efficiency was confirmed by reverse transcription-quantitative PCR and western blot analysis in (A and B) HeLa and (C and D) SiHa cells. CCT3 was inhibited by shRNA in HeLa and SiHa cells. Cell migratory and invasive abilities were validated by Transwell assays. CCT3-knockdown inhibited (E) SiHa and (F) HeLa cell migration as well as (G) $\mathrm{SiHa}$ and $(\mathrm{H}) \mathrm{HeLa}$ cell invasiveness. ${ }^{* *} \mathrm{P}<0.01$ and ${ }^{* * *} \mathrm{P}<0.001$. CCT3, chaperonin containing TCP1 subunit 3; CESC, cervical squamous cell carcinoma and endocervical adenocarcinoma; sh, short hairpin (RNA); Ctrl, control.

CCT3 in tumor progression, CCT3 mRNA expression was first measured in various tumors using the TIMER database. The data revealed that CCT3 expression was increased in multiple types of human cancer compared with healthy individuals (Fig. 1A). Additionally, the analysis of GEPIA datasets revealed that $\mathrm{CCT} 3$ expression was markedly increased in 50 paired CESC tumors (Fig. 1B). The upregulated expression of CCT3 in tumors was further confirmed in the GSE63514, GSE46857 and GSE9750 datasets (Fig. 1C-E). These results indicated that CCT3 may play a role in CESC.

Increased expression of CCT3 is associated with poor patient prognosis. The results of the present study further revealed that in the Protein Atlas database, CCT3 protein expression 

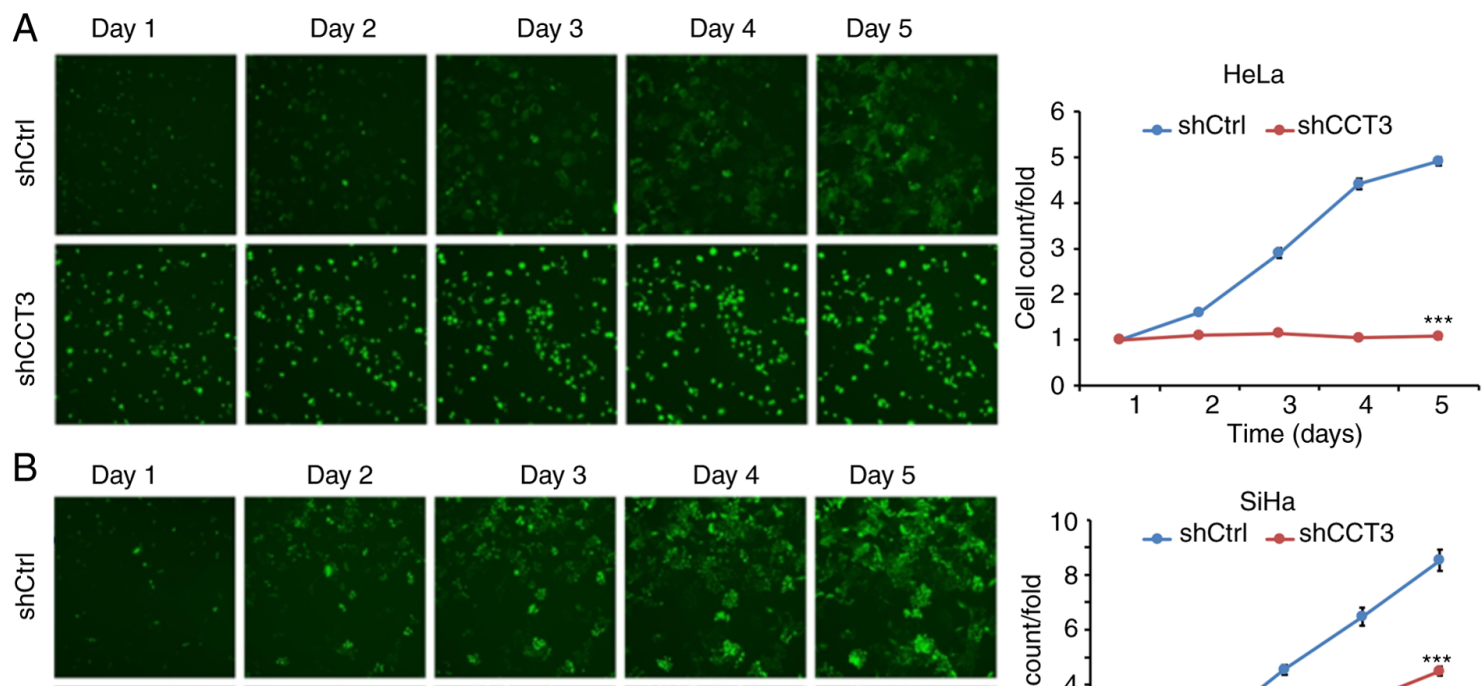

Day 5
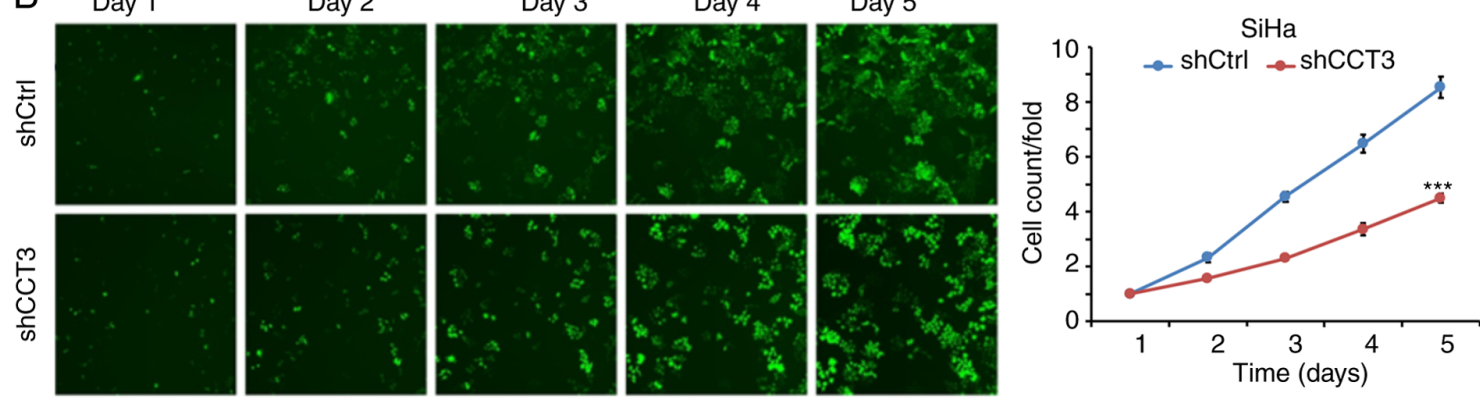

C
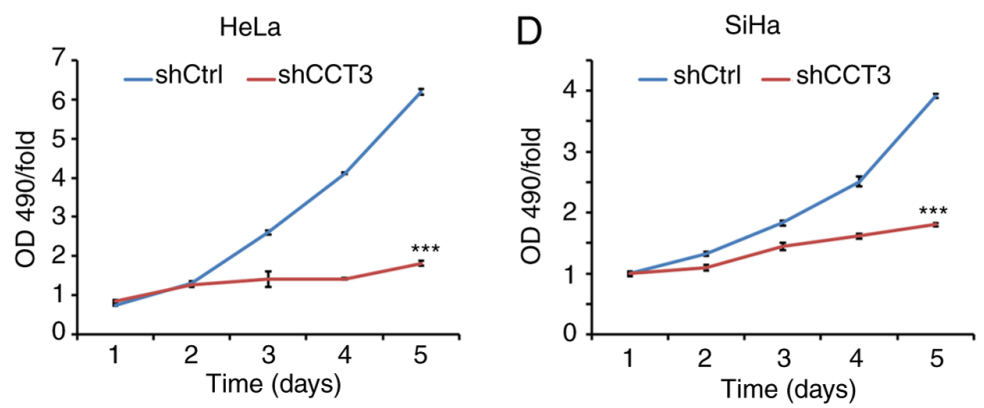

Figure 4. CCT3-knockdown suppresses CESC cell proliferation. CCT3 expression was knocked down using shRNA in (A) HeLa and (B) SiHa cells. Celigo analysis revealed that CCT3-knockdown suppressed the proliferation of both cell lines. MTT analysis revealed that the knockdown of CCT3 suppressed the proliferation of (C) HeLa and (D) SiHa cells. ${ }^{* * *} \mathrm{P}<0.001$ vs. control. CCT3, chaperonin containing TCP1 subunit 3; CESC, cervical squamous cell carcinoma and endocervical adenocarcinoma; sh, short hairpin (RNA); Ctrl, control.

was increased in CESC samples compared with normal samples (Fig. 2A). Furthermore, increased CCT3 expression was associated with a shorter overall survival rate, as indicated by Kaplan-Meier survival using the GEPIA database (Fig. 2B). The results also revealed that a higher expression level of CCT3 was associated with the poor disease-free survival of patients with CESC, as determined using data from the GEPIA database (Fig. 2C). These findings demonstrated that CCT3 may be a potential biomarker for CESC that may be involved in regulating cancer progression.

CCT3-knockdown decreases cellular migration and invasion ability. Next, HeLa and SiHa cells were established in which CCT3 expression was knocked down (Fig. 3). CCT3 mRNA and protein levels were then determined in the HeLa and $\mathrm{SiHa}$ cells transfected with CCT3 shRNA (Fig. 3A-D). The results of the Transwell assay indicated that the CCT3-knockdown inhibited the migration of HeLa and SiHa cells by $\sim 90$ and $70 \%$, respectively (Fig. 3E and F). Subsequently, the effects of CCT3-knockdown on CESC cell invasiveness were evaluated. The results revealed that the invasiveness of HeLa and SiHa cells was decreased by $\sim 90$ and $45 \%$, respectively, in the
CCT3-knockdown groups compared with the control groups (Fig. 3G and H).

CESC cell cycle progression is inhibited, and apoptosis is induced, following CCT3-knockdown. Cellular proliferation analysis using Celigo (Fig. 4A and B) and MTT (Fig. 4C and D) assays demonstrated that CCT3-knockdown significantly inhibited the proliferation of both HeLa and SiHa cells. CCT3 has been reported to participate in cell cycle regulation (22). The present study data revealed that decreased expression of CCT3 increased the proportion of cells in the G2/M phase, but decreased the G0/G1 ratio in SiHa and HeLa cells (Fig. 5A and $\mathrm{B}$, respectively). The results also revealed that $\mathrm{SiHa}$ and HeLa cells with knocked down CCT3 expression exhibited higher levels of apoptosis (Fig. 5C and D). Moreover, expression of the pro-apoptotic protein $\mathrm{Bcl} 2$ was inhibited following CCT3-knockdown, while anti-apoptotic Bax was upregulated in both $\mathrm{SiHa}$ and HeLa cells (Fig. 5E). However, a promotive effect of CCT3 overexpression in cellular proliferation, migration and invasiveness was not observed in $\mathrm{HeLa}$ or $\mathrm{SiHa}$ cells (Fig. 6A-F). However, these results still suggest that CCT3 promoted CESC cell proliferation, migration and invasiveness. 

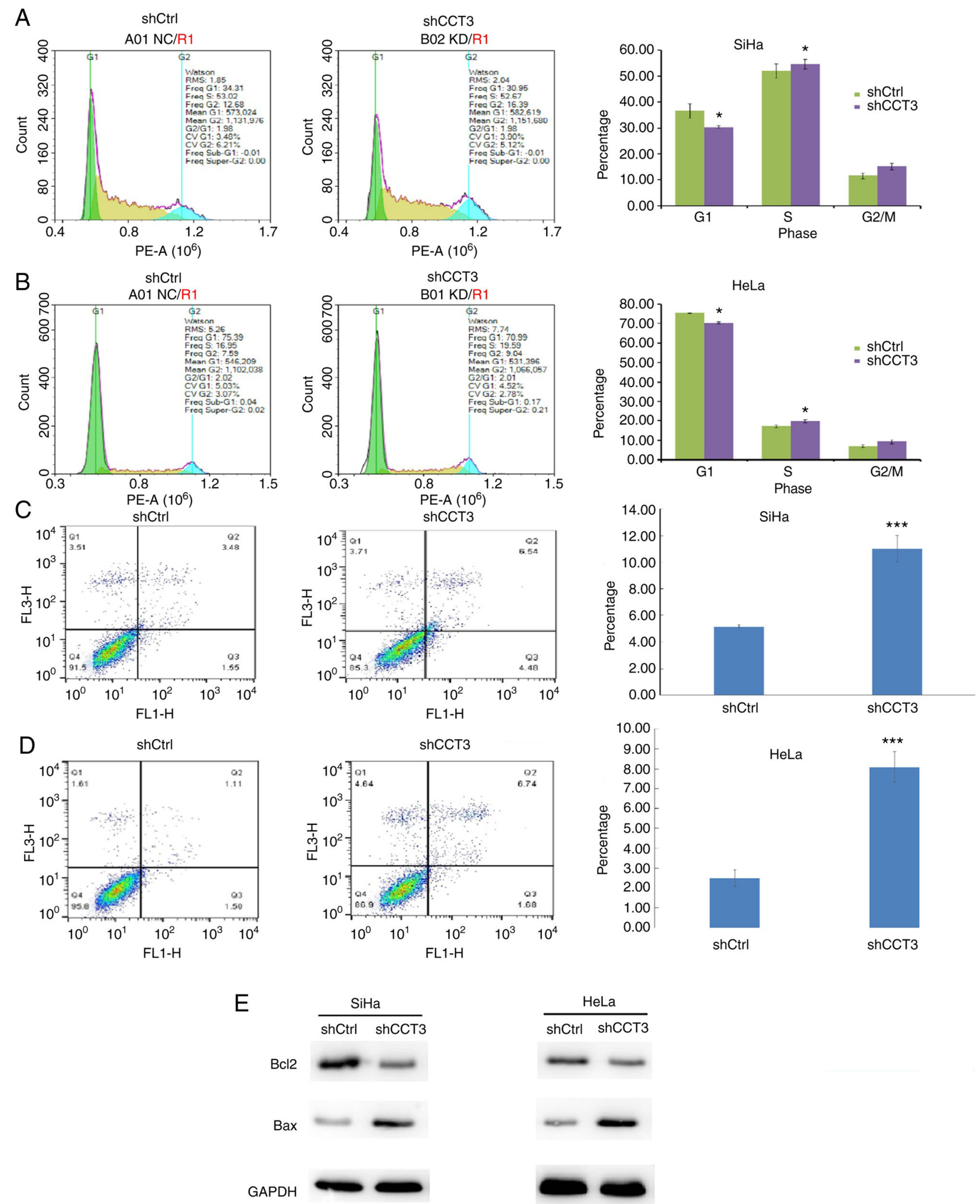

Figure 5. CCT3-knockdown induces cell cycle arrest and apoptosis in CESC cells. CCT3 was inhibited by shRNA in HeLa and SiHa cells. Flow cytometric analysis revealed a significant increase in the proportion of cells in the G2/M phase in (A) SiHa and (B) HeLa cells in which CCT3 expression was knocked down. Silencing of CCT3 also promoted (C) SiHa and (D) HeLa cell apoptosis. (E) Bcl2 and Bax protein expression levels were downregulated and upregulated, respectively, as detected via western blotting. ${ }^{*} \mathrm{P}<0.05$ and ${ }^{* * * *} \mathrm{P}<0.01$ vs. control. CCT3, chaperonin containing TCP1 subunit 3 ; CESC, cervical squamous cell carcinoma and endocervical adenocarcinoma; sh, short hairpin (RNA); Ctrl, control.

CCT3 affects the expression of multiple key cancer regulators in CESC. To evaluate the potential mechanisms of action of CCT3 in CESC, the expression levels of multiple proliferation, migration and cell cycle regulators [including cadherin
$(\mathrm{CDH}) 1, \mathrm{CDH} 2$, phosphorylated (p)-p38, FN1, matrix metalloproteinase (MMP)2, Twist-related protein (TWIST), MMP9, Snail, mTOR, MYC, NF-кB-p65, p-mTOR, vimentin (VIM), p-NF-кB-p65, p- $\beta$-catenin, p38, $\beta$-catenin and Slug] were 
A

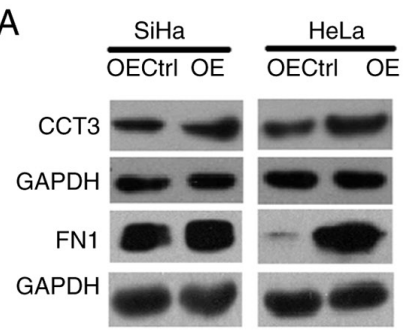

$\mathrm{D}$

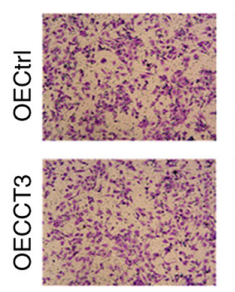

$\mathrm{E}$
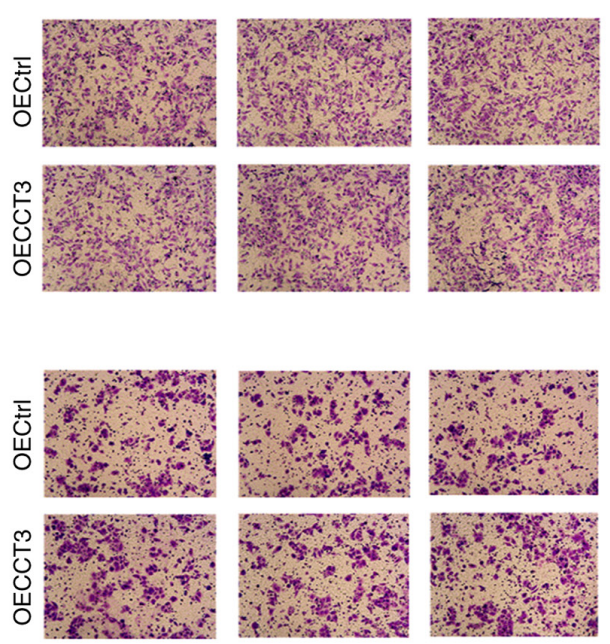

$\mathrm{F}$
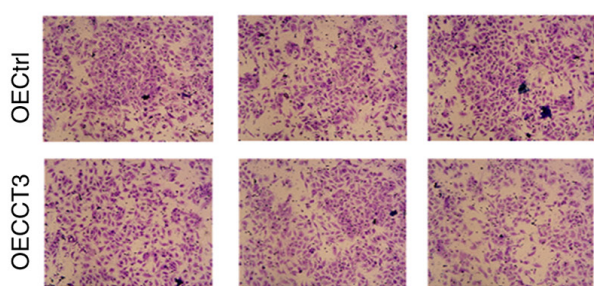

G

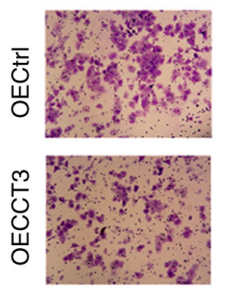

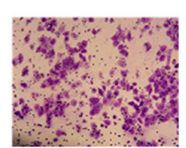
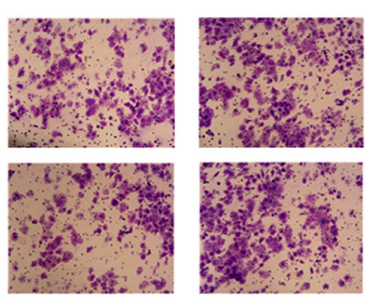
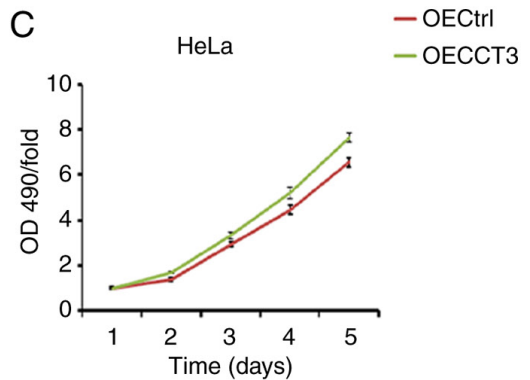

$\mathrm{SiHa}$
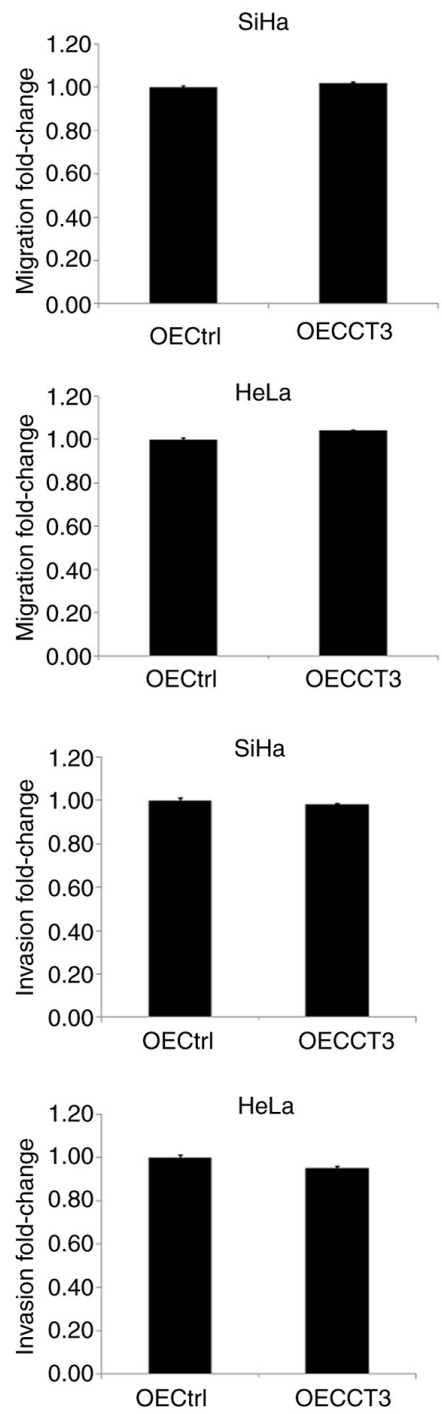

Figure 6. Effects of CCT3 overexpression on CESC. (A) CCT3 and FN1 were overexpressed in SiHa and HeLa cells. MTT assays demonstrated that the overexpression of CCT3 did not significantly induce proliferation rate of (B) SiHa or (C) HeLa cells. Cell migratory and invasive abilities were validated by Transwell assays. Overexpression of CCT3 in (D) SiHa and (E) HeLa cell lines did not significantly later cell migration. Overexpression of CCT3 in (F) SiHa and (G) HeLa cell lines did not significantly induce cell invasiveness. CCT3, chaperonin containing TCP1 subunit 3; FN1, fibronectin 1; CESC, cervical squamous cell carcinoma and endocervical adenocarcinoma; OE, overexpression; Ctrl, control.

detected in SiHa cells following CCT3-knockdown. The results revealed that the protein levels of p-p38, FN1 and MMP9 were markedly downregulated following CCT3-knockdown (Fig. 7A). Among these proteins, FN1 was selected for further validation as other downstream proteins were unable to rescue CCT3 knockdown-induced cell proliferative inhibition (data not shown). Of note, CCT3 expression was significantly and positively correlated with that of FN1 in CESC (Fig. 7B). A higher expression level of FN1 was also associated with a shorter disease-free survival time in CESC (Fig. 7C and D). These results suggested that FN1 may function as a downstream regulator of CCT3 in human CESC.

Overexpression of FN1 rescues the effects on CESC mediated by CCT3-knockdown. The present study further investigated the association between CCT3 and FN1. Cell viability assays revealed that the overexpression of FN1 significantly reversed the suppressive effects of CCT3-knockdown on the proliferation of both 

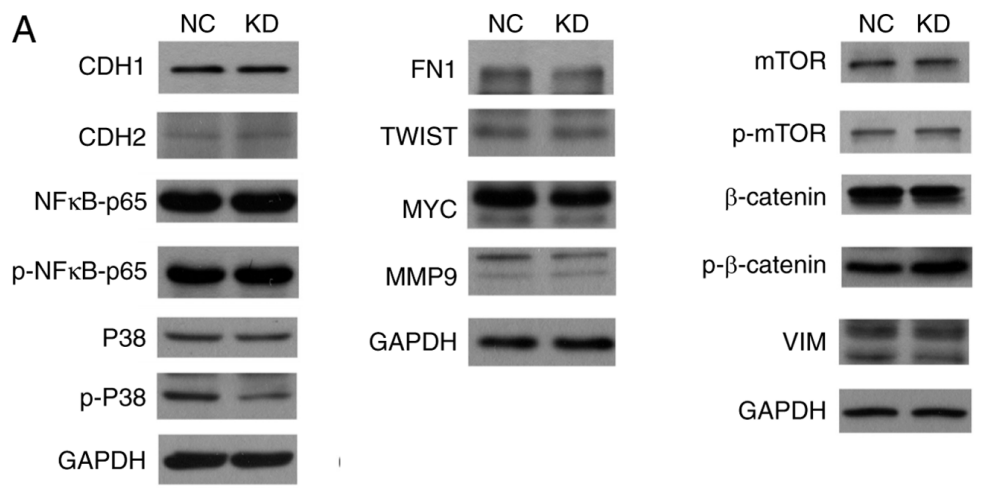

B
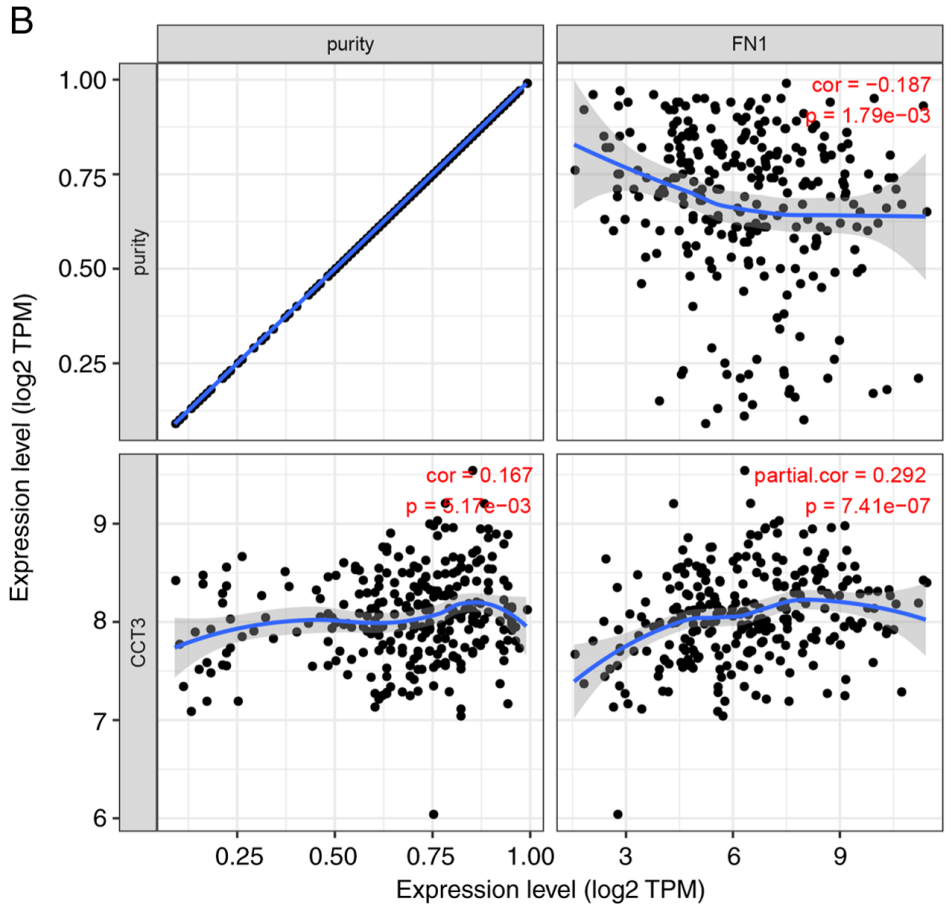

C
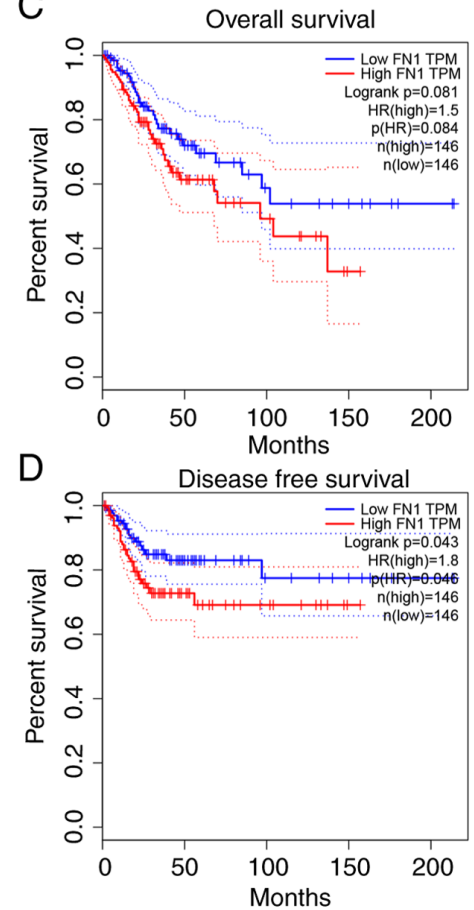

Figure 7. CCT3-knockdown suppresses FN1 expression. (A) Protein expression levels of CDH1, CDH2, p-p38, FN1, MMP2, TWIST, MMP9, Snail, mTOR, MYC, NF-кB-p65, p-mTOR, VIM, p-NF-кB-p65, p- $\beta$-catenin, p38, $\beta$-catenin and Slug were detected following CCT3-knockdown in SiHa cells. (B) CCT3 expression was significantly positively correlated with FN1 expression in CESC. (C and D) Higher expression of CCT3 was associated with a shorter disease-free survival time, but not with overall survival time in CESC. CCT3, chaperonin containing TCP1 subunit 3; CESC, cervical squamous cell carcinoma and endocervical adenocarcinoma; $\mathrm{CDH} 1$, cadherin 1; $\mathrm{CDH}$ 2, cadherin 2; FN1, fibronectin 1; MMP2, matrix metalloproteinase 2; TWIST, Twist-related protein; MMP9, matrix metalloproteinase 9; VIM, vimentin; p-, phosphorylated; NC, negative control; KD, knockdown; OE, overexpression.

HeLa and SiHa cells (Fig. 8A-D). Furthermore, the results of the Transwell assays revealed that the overexpression of FN1 significantly reversed the suppressive effects of CCT3-knockdown on the migration of SiHa and HeLa cells (Fig. 8E). Collectively, these data suggested that the regulatory effects of CCT3 on CESC cell functions were mediated via the FN1 gene.

The present study identified that CCT3 expression levels were upregulated in CESC tissues, which was associated with poor patient prognosis. Moreover, the findings revealed that CCT3 suppressed apoptosis and promoted cell cycle progression and metastatic capacity via the FN1 signaling pathway, which demonstrated that CCT3 may prove to be a potential biomarker for CESC.

\section{Discussion}

CESC is a major cause of cancer-related mortality among women in China (2). However, the mechanisms regulating the development of CESC remain largely unknown. It is thus imperative to investigate the underlying mechanisms, and to identify promising prognostic indicators, for CESC. The results of the present study revealed that CCT3 expression levels were higher in CESC tissues, and that this was associated with a poor prognosis.

CCT proteins have been demonstrated to play an elementary role in human cancers, including breast cancer and lung cancer, and multiple members of this family have been found to be abnormally expressed, and to be associated with tumor development (7,23-25). For example, CCT2 expression was found to be upregulated in liver (26), colon (10) and lung cancer (27). Higher CCT2 levels are also associated with shorter overall survival times in patients with non-small cell lung cancer (27). CCT8 expression has been revealed to be upregulated in HCC and glioma, and to promote cellular proliferation and migration therein (28). Furthermore, CCT3 expression was found to be upregulated in liver, breast cancer 
A

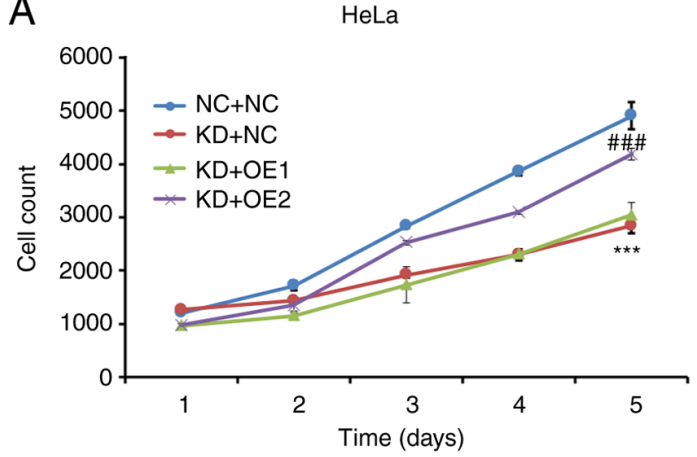

C

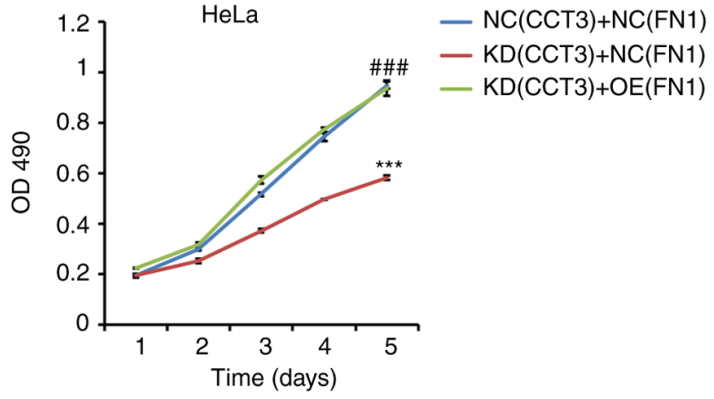

B

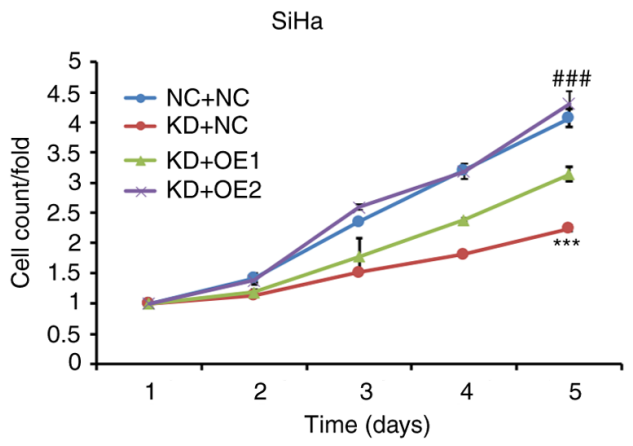

D

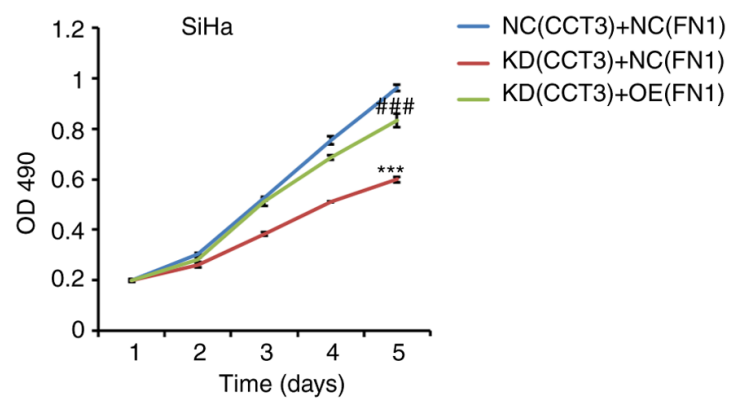

$\mathrm{E}$
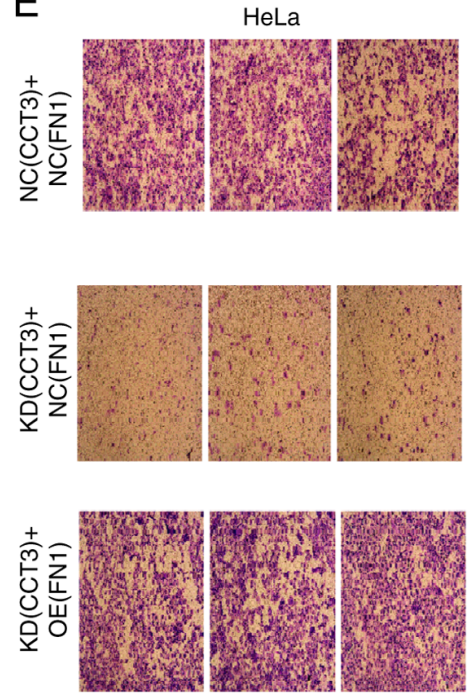

$\mathrm{SiHa}$
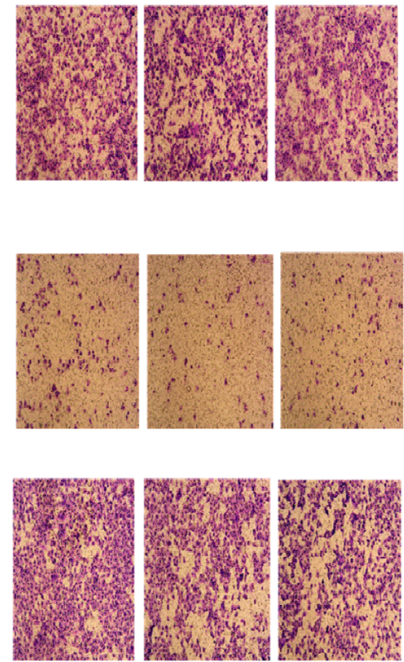

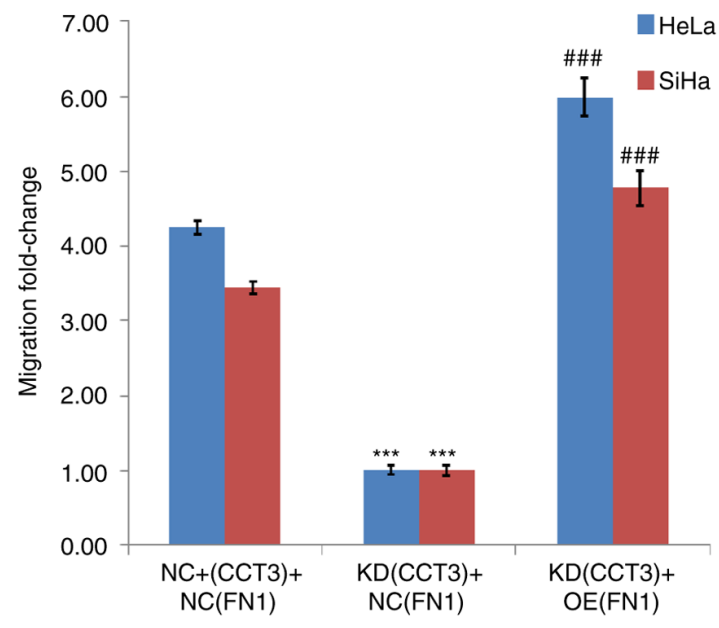

Figure 8. Overexpression of FN1 reverses the effects of CCT3-knockdown on CESC cells. Celigo analysis revealed that the overexpression of FN1 increased the proliferation rate of (A) HeLa and (B) SiHa cells following CCT3-knockdown. MTT assays revealed that the overexpression of FN1 increased the proliferation rate of (C) HeLa and (D) SiHa cells following CCT3-knockdown. (E) Transwell analysis revealed that the overexpression of FN1 increased HeLa and SiHa cell migration rate, following CCT3-knockdown. ${ }^{* * *} \mathrm{P}<0.001$ (CCT3 KD + FN1 ND vs. CCT3 NC + FN1 NC); \#\# $<0.001$ (CCT3 KD + FN1 OE vs. CCT3 KD + FN1 OE). CCT3, chaperonin containing TCP1 subunit 3; CESC, cervical squamous cell carcinoma and endocervical adenocarcinoma; FN1, fibronectin 1; $\mathrm{NC}$, negative control; KD, knockdown.

and colorectal cancer $(22,26,29)$. To the best of our knowledge, the present study was the first to detect the expression patterns and prognostic value of CCT3 in CESC using TCGA and GEO databases, where CCT3 expression was found to be upregulated in tumor samples compared with normal tissues.

Bioinformatics analysis also confirmed CCT3 as a tumor promoter in CESC cell models. Kaplan-Meier survival and Cox proportional hazards regression analyses indicated that CCT3 expression was associated with the shorter survival time of patients with CESC. Furthermore, in vitro studies indicated that CCT3 promoted the metastatic capacity of tumor cells; CCT3-knockdown significantly reduced the migration and invasiveness of CESC cells. However, when CCT3 was overexpressed, limited increases in CESC cell proliferation and migration were observed. This may be attributed to the fact that high expression of background CCT3 (or exogenous upregulation) in CESC cells is sufficient for promoting cellular proliferation and migration. In previous studies, CCT3 was found to be a regulator of the cell cycle in multiple cancer types $(15,22,26)$. Thus, the present study further validated the potential effects of CCT3-knockdown, demonstrating a marked suppression in CESC cell proliferation and cell cycle progression, and apoptosis promotion. After screening multiple cancer regulators, FN1 expression levels were downregulated 
when CCT3 was inhibited. Moreover, FN1 overexpression rescued the inhibitory effect of CCT3. These data identified that FN1 may be a downstream target of CCT3. To the best of our knowledge, the present study demonstrated for the first time that CCT3 promoted FN1 expression in CESC cells.

FN1 is a member of the FN protein family, is widely expressed in multiple cell types, and plays pivotal roles in cellular adhesion and migration processes (30). Previous research has demonstrated that higher expression levels of FN1 are associated with advanced tumor stage (31). FN1 has been reported to be involved in tumor metastasis and extracellular matrix-related changes, such as the epithelial-mesenchymal transition (32). The downregulation of FN1 has also been shown to suppress colorectal tumorigenesis by regulating proliferation, migration and invasion (33), and emerging evidence has indicated that FN1 is an important regulator of tumor cell chemoresistance (34). In addition, mechanistic analyses have suggested that FN1 may interact with vascular endothelial growth factor A, and play a primary role in non-small cell lung cancer (35). Moreover, FN1 was reported to promote Src and caspase- 8 phosphorylation in lung cancer cells. The present study identified multiple proliferation, migration and cell cycle regulators in $\mathrm{SiHa}$ cells following CCT3-knockdown, including CDH1, CDH2, p-p38, FN1, MMP2, TWIST, MMP9, mTOR, MYC, NF-кB-p65, p-mTOR, VIM, p-NF- $\mathrm{B}$-p65, p- $\beta$-catenin, p38 and $\beta$-catenin. FN1 protein levels were suppressed following CCT3-knockdown in CESC cells, and thus, FN1 may be a downstream target of CCT3. FN1 was subsequently selected for further validation. However, this does not mean that there are no other proteins of research value in this context, such as p53, retinoblastoma or PTEN $(36,37)$. Furthermore, cell viability and Transwell assays revealed that the overexpression of FN1 significantly reversed the suppressive effects of CCT3-knockdown on the proliferation and migration of $\mathrm{HeLa}$ and $\mathrm{SiHa}$ cells. Collectively, these data suggest that CCT3 induced its effects on cellular functions via regulation of the FN1 gene.

In conclusion, the results of the present study indicated that CCT3 acts as an oncogene in CESC, and was found to be associated with a poor patient prognosis. The data also indicated that CCT3 promoted cellular invasion and migration ability. Moreover, CCT3 was found to be involved in regulating FN1 gene expression, thus regulating cell cycle and migration progression. Collectively, the data indicate that CCT3 may be regarded as a prognostic indicator of CESC, and may prove to be a novel target for the treatment of CESC.

\section{Acknowledgements}

Not applicable.

\section{Funding}

The present study was supported by internal funding from The First Hospital of China Medical University (Shenyang, China).

\section{Availability of data and materials}

The datasets used and/or analyzed during the current study are available from the corresponding author on reasonable request.

\section{Authors' contributions}

LD designed the experiments and wrote the paper. XZ performed the experiments and participated in study design and writing. Both authors have read and approved the manuscript. LD and XZ confirm the authenticity of all the raw data.

\section{Ethics approval and consent to participate}

Not applicable.

\section{Patient consent for publication}

Not applicable.

\section{Competing interests}

The authors declare that they have no competing interests

\section{References}

1. Torre LA, Bray F, Siegel RL, Ferlay J, Lortet-Tieulent J and Jemal A: Global cancer statistics, 2012. CA Cancer J Clin 65: 87-108, 2015

2. Zhang X, Li F and Zhu L: Clinical significance and functions of microRNA-93/CDKN1A axis in human cervical cancer. Life Sci 209: 242-248, 2018.

3. Zhu X, Zhou L, Li R, Shen Q, Cheng H, Shen Z and Zhu H: AGER promotes proliferation and migration in cervical cancer. Biosci Rep 38: BSR20171329, 2018.

4. Zhu H, Shen Z, Luo H, Zhang W and Zhu X: Chlamydia Trachomatis Infection-Associated Risk of Cervical Cancer: A Meta-Analysis. Medicine (Baltimore) 95: e3077, 2016.

5. Zhu H, Luo H, Shen Z, Hu X, Sun L and Zhu X: Transforming growth factor- $\beta 1$ in carcinogenesis, progression, and therapy in cervical cancer. Tumour Biol 37: 7075-7083, 2016.

6. Yang Z, Sun Q, Guo J, Wang S, Song G, Liu W, Liu M and Tang H: GRSF1-mediated MIR-G-1 promotes malignant behavior and nuclear autophagy by directly upregulating TMED5 and LMNB1 in cervical cancer cells. Autophagy 15: 668-685, 2019.

7. Lin YF, Tsai WP, Liu HG and Liang PH: Intracellular beta-tubulin/chaperonin containing TCP1-beta complex serves as a novel chemotherapeutic target against drug-resistant tumors. Cancer Res 69: 6879-6888, 2009.

8. Vallin $\mathrm{J}$ and Grantham J: The role of the molecular chaperone CCT in protein folding and mediation of cytoskeleton-associated processes: Implications for cancer cell biology. Cell Stress Chaperones 24: 17-27, 2019.

9. Klimczak M, Biecek P, Zylicz A and Zylicz M: Heat shock proteins create a signature to predict the clinical outcome in breast cancer. Sci Rep 9: 7507, 2019.

10. Park SH, Jeong S, Kim BR, Jeong YA, Kim JL, Na YJ, Jo MJ, Yun HK, Kim DY, Kim BG, et al: Activating CCT2 triggers Gli-1 activation during hypoxic condition in colorectal cancer. Oncogene 39: 136-150, 2020.

11. Wang L, Zhao H, Li J, Xu Y, Lan Y, Yin W, Liu X, Yu L, Lin S, Du MY, et al: Identifying functions and prognostic biomarkers of network motifs marked by diverse chromatin states in human cell lines. Oncogene 39: 677-689, 2020.

12. Liu P, Kong L, Jin H, Wu Y, Tan X and Song B: Differential secretome of pancreatic cancer cells in serum-containing conditioned medium reveals CCT8 as a new biomarker of pancreatic cancer invasion and metastasis. Cancer Cell Int 19: 262, 2019.

13. Liu Y, Zhang X, Lin J, Chen Y, Qiao Y, Guo S, Yang Y, Zhu G, Pan Q, Wang J, et al: CCT3 acts upstream of YAP and TFCP2 as a potential target and tumour biomarker in liver cancer. Cell Death Dis 10: 644, 2019.

14. Qian EN, Han SY, Ding SZ and Lv X: Expression and diagnostic value of CCT3 and IQGAP3 in hepatocellular carcinoma. Cancer Cell Int 16: 55, 2016.

15. Shi X, Cheng S and Wang W: Suppression of CCT3 inhibits malignant proliferation of human papillary thyroid carcinoma cell. Oncol Lett 15: 9202-9208, 2018. 
16. Cui F, Hu J, Fan Y, Tan J and Tang H: Knockdown of spindle pole body component 25 homolog inhibits cell proliferation and cycle progression in prostate cancer. Oncol Lett 15: 5712-5720, 2018.

17. Wan X, Pu H, Huang W, Yang S, Zhang Y, Kong Z, Yang Z, Zhao P, Li A, Li T, et al: Androgen-induced miR-135a acts as a tumor suppressor through downregulating RBAK and MMP11, and mediates resistance to androgen deprivation therapy. Oncotarget 7: 51284-51300, 2016.

18. Livak KJ and Schmittgen TD: Analysis of relative gene expression data using real-time quantitative PCR and the 2(-Delta Delta C(T)) Method. Methods 25: 402-408, 2001.

19. den Boon JA, Pyeon D, Wang SS, Horswill M, Schiffman M, Sherman M, Zuna RE, Wang Z, Hewitt SM, Pearson R, et al: Molecular transitions from papillomavirus infection to cervical precancer and cancer: Role of stromal estrogen receptor signaling. Proc Natl Acad Sci USA 112: E3255-E3264, 2015.

20. Thomas A, Mahantshetty U, Kannan S, Deodhar K, Shrivastava SK, Kumar-Sinha C and Mulherkar R: Expression profiling of cervical cancers in Indian women at different stages to identify gene signatures during progression of the disease. Cancer Med 2: 836-848, 2013.

21. Scotto L, Narayan G, Nandula SV, Arias-Pulido H, Subramaniyam S, Schneider A, Kaufmann AM, Wright JD, Pothuri B, Mansukhani M, et al: Identification of copy number gain and overexpressed genes on chromosome arm $20 \mathrm{q}$ by an integrative genomic approach in cervical cancer: Potential role in progression. Genes Chromosomes Cancer 47: 755-765, 2008.

22. Xu G, Bu S, Wang X, Zhang H and Ge H: Suppression of CCT3 inhibits the proliferation and migration in breast cancer cells Cancer Cell Int 20: 218, 2020.

23. Dun MD, Smith ND, Baker MA, Lin M, Aitken RJ and Nixon B: The chaperonin containing TCP1 complex (CCT/TRiC) is involved in mediating sperm-oocyte interaction. J Biol Chem 286: 36875-36887, 2011

24. Nadler-Holly M, Breker M, Gruber R, Azia A, Gymrek M, Eisenstein M, Willison KR, Schuldiner M and Horovitz A: Interactions of subunit CCT3 in the yeast chaperonin CCT/TRiC with $\mathrm{Q} / \mathrm{N}$-rich proteins revealed by high-throughput microscopy analysis. Proc Natl Acad Sci USA 109: 18833-18838, 2012.

25. Syed A, Lukacsovich T, Pomeroy M, Bardwell AJ, Decker GT, Waymire KG, Purcell J, Huang W, Gui J, Padilla EM, et al: Miles to go (mtgo) encodes FNDC3 proteins that interact with the chaperonin subunit CCT3 and are required for NMJ branching and growth in Drosophila. Dev Biol 445: 37-53, 2019.

26. Yao L, Zou X and Liu L: The TCP1 ring complex is associated with malignancy and poor prognosis in hepatocellular carcinoma. Int J Clin Exp Pathol 12: 3329-3343, 2019.

27. Carr AC, Khaled AS, Bassiouni R, Flores O, Nierenberg D, Bhatti H, Vishnubhotla P, Manuel JP, Santra S and Khaled AR: Targeting chaperonin containing TCP1 (CCT) as a molecular therapeutic for small cell lung cancer. Oncotarget 8: 110273-110288, 2017.
28. Qiu X, He X, Huang Q, Liu X, Sun G, Guo J, Yuan D, Yang L, Ban N, Fan S, et al: Overexpression of CCT8 and its significance for tumor cell proliferation, migration and invasion in glioma. Pathol Res Pract 211: 717-725, 2015.

29. Nibbe RK, Markowitz S, Myeroff L, Ewing R and Chance MR Discovery and scoring of protein interaction subnetworks discriminative of late stage human colon cancer. Mol Cell Proteomics 8: 827-845, 2009.

30. Wang J, Deng L, Huang J, Cai R, Zhu X, Liu F, Wang Q, Zhang J and Zheng Y: High expression of Fibronectin 1 suppresses apoptosis through the NF- $\mathrm{BB}$ pathway and is associated with migration in nasopharyngeal carcinoma. Am J Transl Res 9: 4502-4511, 2017.

31. Li B, Shen W, Peng H, Li Y, Chen F, Zheng L, Xu J and Jia L: Fibronectin 1 promotes melanoma proliferation and metastasis by inhibiting apoptosis and regulating EMT. OncoTargets Ther 12: 3207-3221, 2019.

32. Soikkeli J, Podlasz P, Yin M, Nummela P, Jahkola T, Virolainen S, Krogerus L, Heikkilä P, von Smitten K, Saksela O, et al: Metastatic outgrowth encompasses COL-I, FN1, and POSTN up-regulation and assembly to fibrillar networks regulating cell adhesion, migration, and growth. Am J Pathol 177: 387-403, 2010.

33. Cai X, Liu C, Zhang TN, Zhu YW, Dong X and Xue P: Down-regulation of FN1 inhibits colorectal carcinogenesis by suppressing proliferation, migration, and invasion. J Cell Biochem 119: 4717-4728, 2018.

34. Yang X, Hu Q, Hu LX, Lin XR, Liu JQ, Lin X, Dinglin XX, Zeng JY, Hu H, Luo ML, et al: miR-200b regulates epithelial-mesenchymal transition of chemo-resistant breast cancer cells by targeting FN1. Discov Med 24: 75-85, 2017.

35. Wang Y, Huang L, Wu S, Jia Y, Yang Y, Luo L, Bi A and Fang M: Bioinformatics analyses of the role of vascular endothelial growth factor in patients with non-small cell lung cancer. PLoS One 10: e0139285, 2015.

36. Balasubramaniam SD, Balakrishnan V, Oon CE and Kaur G: Key molecular events in cervical cancer development. Medicina (Kaunas) 55: 384, 2019.

37. Nero C, Ciccarone F, Pietragalla A and Scambia G: PTEN and Gynecological cancers. Cancers (Basel) 11: 1458, 2019.

This work is licensed under a Creative Commons Attribution-NonCommercial-NoDerivatives 4.0 International (CC BY-NC-ND 4.0) License. 